\title{
Comparing the enhanced sensing interfaces of differently oriented carbon nanotubes onto silicon for bio-chip applications
}

\author{
Irene Taurino* ${ }^{* \dagger}$, Sandro Carrara*, Mauro Giorcelli ${ }^{\dagger}$, Alberto Tagliaferro ${ }^{\dagger}$ and Giovanni De Micheli* \\ * EPFL - École Polytechnique Fédérale de Lausanne - CH - Lausanne \\ $\dagger$ Politecnico di Torino - IT - Torino \\ Email: sandro.carrara@epfl.ch
}

\begin{abstract}
Carbon nanotubes improve the sensitivity of electrochemical biochips. An optimized integration of the nano/bio/CMOS interface is required to realize accurate and low-cost sensors for applications in personalized medicine. The nanotubes orientation on the chip is a key parameter. The role of the sidewalls and the tips surface on the electro-activity of the nanosensor is still subject of debate in the literature. In this paper, a comparison between vertical densely-packed and randomlyoriented carbon nanotubes directly growth on silicon wafer is proposed in order to identify the best nano-system for biosensing purposes. The comparison is done by using contact-angle measurements, energy dispersive $\mathrm{X}$-ray analysis and electrochemical voltammetry.
\end{abstract}

\section{INTRODUCTION}

The design of accurate, low-cost, portable, easy-to-use, point-of-care devices for personalized medicine requires novel fundamental and experimental research on circuits and systems [1]. Specific bio-probes - typically proteins or DNA oligos are required to create sensors for electrochemical detection in bio-medical applications, typically in diagnostics.

Carbon Nanotubes (CNTs) are key building blocks to improve the sensing performances of typical electrochemical techniques [2]. Indeed, while standard, non nano-structured electrodes provide good sensitivity only in millimolar (mM) ranges [3], nano-structured electrodes using multi walled CNTs (MWCNTs) reach detection limits enabling sensing in the micromolar $(\mu \mathrm{M})$ range, that is the physiological concentration range of target metabolites in human serum [4]. Dedicated CMOS circuits are definitely required to address special spots query in order to improve targets identification and quantification [1]. Therefore, optimized nano/bio/CMOS interfaces and circuits are crucial to design accurate and low-cost devices for detecting endogenous biomarkers as well as exogenous drugs that are commonly used in well-known therapy for patients'treatment.

In this paper, we focus on the improved sensor performances of differently-oriented MWCNTs directly growth onto silicon wafers with the aim to identify the best orientation and chemical treatments that can optimize the electrochemical sensing.

The electrochemical performance of CNT-based electrodes depends on many factors, like synthesis methods [5], surface treatments [6], bounding with the substrate [7], [2], orientation of tubes and type of targets [8]. Most of the literature on CNTs is based on the assumption that tips are responsible for their electrochemical activity. The role of the sidewall is still under investigation [9]. However, recent studies demonstrate a large contribution from the sidewalls in case of randomly oriented CNTs [9]. They generate alterations in nanotube sidewalls changing the electrochemical properties [10].

The aim of this work is to investigate the improved performance of a CNT-based bio-chip by investigating the electrochemical characteristics of randomly and dense vertically oriented MWCNTs before and after acid treatment. CNTs are fabricated via chemical vapor deposition (CVD) directly on a silicon wafer. Contact angles measurements prove the effects of the treatment. Field-emission scanning electron microscopy (FE-SEM) images and energy dispersive $X$-ray (EDX) spectra of pristine and treated MWCNTs are acquired to observe possible compositional (oxygen-contained groups) and morphological changes related to acid treatment. Electrochemical studies are preformed by using cyclic voltammetry. This investigation is carried out to improve nano/CMOS integration for VLSI design for a bio-chip based on CNTs.

\section{MATERIALS AND METHODS}

\section{A. Fabrication of MWCNTs onto silicon wafer}

MWCNTs are grown onto silicon wafer (Si-mat, Germany) via CVD. Camphor and ferrocene are used respectively as carbon precursor and catalyst. Commercial camphor is selected as carbon precursor, because its 3D structure helps the formation of nanotube rings [11]. Moreover, camphor is non-toxic for human health and for the environment [12], [13]. Ferrocene (98\% purity in weight, Aldrich) is an organometallic chemical compound acting as both a catalyst and a carbon source. It is the source of iron atoms that agglomerate in clusters on which CNTs grow. We utilize a CVD reactor consisting of a furnace fed via two evaporation chambers where the precursors are heated. The silicon substrate is located on a graphite plate and is heated up at the desired temperature $\left(700^{\circ} \mathrm{C}-925^{\circ} \mathrm{C}\right)$. By varying the deposition conditions different structures are obtained. 
- To obtain random MWCNTs, the substrate is heated at $700^{\circ} \mathrm{C}$, ferrocene is introduced in the deposition chamber for $60 \mathrm{~s}$, and the substrate holder rotates at $3 \mathrm{rms}$.

- Densely packed vertical MWCNTs are obtained with ferrocene introduction of $180 \mathrm{~s}$. Ferrocene is carried in the deposition chamber by a laminar flow of nitrogen $(50$ $\mathrm{cm}^{3} / \mathrm{s}$ ). The substrate is kept at $850^{\circ} \mathrm{C}$ and its holder rotates at $12 \mathrm{rms}$.

In both cases camphor is flown for 10 minutes with no carrier gas. After deposition, the substrate is cooled to room temperature inside the chamber in inert atmosphere.

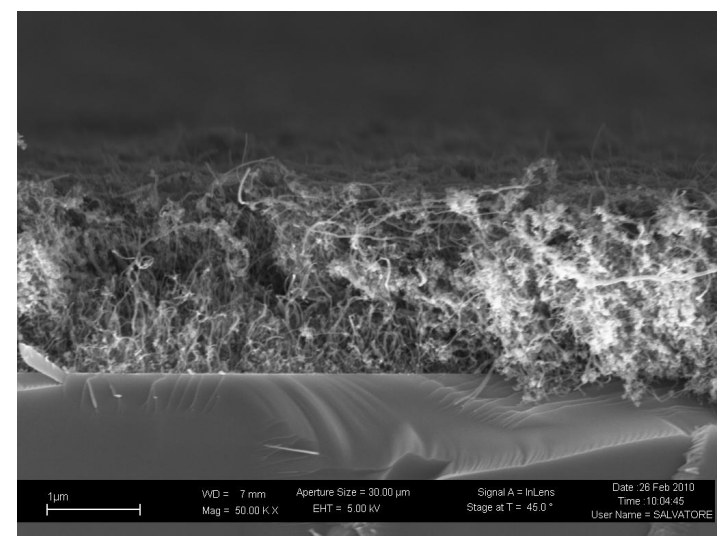

(a)

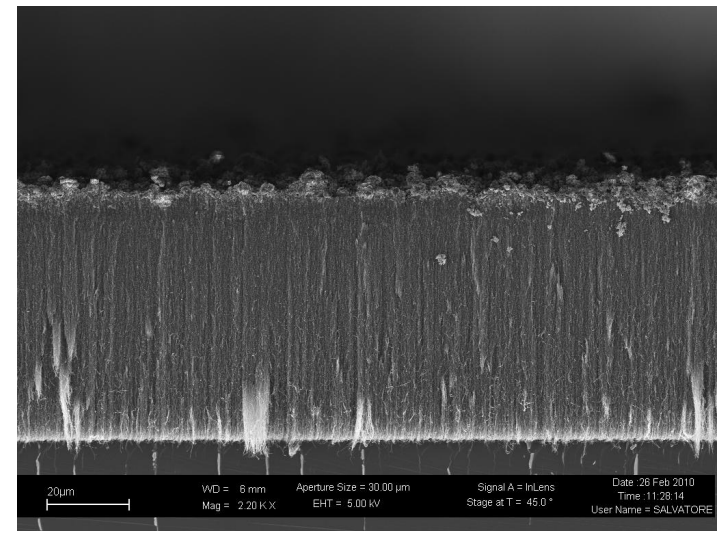

(b)

Fig. 1. FE-SEM images of randomly (a) and dense vertically (b) oriented MWCNTs.

\section{B. Chemicals}

All solutions are prepared using 0.01 M Phosphate Buffer Saline (PBS, Sigma) at pH 7.4. Pristine MWCNTs are treated in $6 \mathrm{M} \mathrm{H}_{2} \mathrm{SO}_{4}$ (Sigma, 95-98\% vol) solution for 6 hours [14]. Potassium ferricyanide in form of powder and hydrogen peroxide $(30 \% \mathrm{vol})$ are purchased from Sigma.

\section{Electrochemical measurements}

Cyclic voltammetry (CV) is performed using Versastat 3 potentiostat (Princeton Applied Technologies) with a stan- dard three electrode configuration. A platinum wire serves as counter electrode, while a wire in $\mathrm{Ag} \mid \mathrm{AgCl}$ saturated with $\mathrm{KCl}(3 \mathrm{M})$ is used as reference electrode (Roschi Rohde and Schwarz AG, Switzerland). MWCNTs on silicon substrate are used as working electrode. All experiments are carried out under aerobic conditions at room temperature.

$\mathrm{CV}$ is performed in solutions with different concentrations of ferricyanide. Potassium ferricyanide couple is chosen as analyte due to the simple and well defined response at carbon materials. The redox reaction at $+300 \mathrm{mV}$ is:

$$
\left[\mathrm{Fe}(\mathrm{CN})_{6}\right]^{-3}+e^{-} \rightarrow\left[\mathrm{Fe}(\mathrm{CN})_{6}\right]^{-4}
$$

The electroactive area $(A)$ is obtained from the slope of anodic peak $\left(I_{p a}\right)$ versus the square root of the scan rate $(\sqrt{v})$ referring to the Rangles-Servic equation:

$$
I_{p}=2.69 \cdot 10^{2} A D^{1 / 2} n^{3 / 2} v^{1 / 2} C
$$

where $I_{p}$ is the current peak, $A$ is the electroactive area, $D$ is the diffusion coefficient of the analyte, $n$ is the number of electrons involved in the redox reaction, $v$ is the scan rate and $C$ is the concentration of the analyte in solution. In particular, ferricyanide couple exhibits one-electron transfer $(n=1)$. The diffusion coefficient $D$ is $5.5 \cdot 10^{-6} \mathrm{~cm} \mathrm{~s}^{-2}$ and ferricyanide concentration is $25 \mathrm{mM}$ for densely vertical and $2.5 \mathrm{mM}$ for randomly oriented MWCNTs. Detection limit is three time signal-to-noise ratio. For ferricyanide, we have varied concentrations at a scan rate of $100 \mathrm{mV} / \mathrm{s}$. The potential is cycled between -0.3 and $+0.7 \mathrm{~V}$ for random MWCNTs, and from -0.2 to $+0.6 \mathrm{~V}$ for vertical aligned nanotubes. The electron transfer rate constant $\left(k_{e t}\right)$ is calculated by varying the scan rate over the range $25-200 \mathrm{mV} / \mathrm{s}$.

$\mathrm{CV}$ is also performed in solutions with different concentrations of $\mathrm{H}_{2} \mathrm{O}_{2}$ because its importance for biosensing applications. It is selected since it is the product of biosensing based on oxidases [9]. A potential of $+650 \mathrm{mV}$ causes the oxidation of hydrogen peroxide, according to the reaction:

$$
2 \mathrm{H}_{2} \mathrm{O}_{2} \rightarrow 2 \mathrm{H}_{2} \mathrm{O}+\mathrm{O}_{2}^{+}+4 e^{-}
$$

Using hydrogen peroxide as target, we have varied concentrations from 0 to $30 \mathrm{mM}$ at a scan rate of $100 \mathrm{mV} / \mathrm{s}$, in order to calculate sensitivity and detection limit. The potential is cycled between -1 and $+1 \mathrm{~V}$.

\section{FE-SEM, EDX and contact angle}

A SUPRA ${ }^{\mathrm{TM}} 40$ (ZEISS) is used to acquire FE-SEM images. Nominal resolution is $1.5 \mathrm{~nm}$ at $10 \mathrm{kV}$.

A Philips/FEI XL-30 F microscope (Netherlands) was used for the EDX analysis. The resolution in UHR mode is $2.5 \mathrm{~nm}$ at $1 \mathrm{kV}$.

Contact angles are measured by using PBS drops $(20 \mu \mathrm{l})$ casted onto the surface of MWCNT-based electrodes, before and after the treatment. The images are acquired with a digital camera. For each sample, the average of five measurements is taken from five different images. 


\section{RESULTS AND DISCUSSION}

\section{A. CNTs fabrication}

Fig. 1 (a) and (b) depict the FE-SEM images obtained for randomly and dense vertically oriented MWCNTs respectively. Randomly oriented MWCNTs (Fig. 1 (a)) are not densely packed. Both walls and tips are exposed to the acid activation. On the other hand, edge ends can be subjected to the treatment by using the "carpet" of vertically oriented MWCNTs (Fig. 1 (b)). FE-SEM images, taken before and after acid activation, do not show morphological changes (data not shown) demonstrating the robustness of our chip fabrications.

\section{B. Contact angle}

Pristine MWCNTs show a hydrophobic behavior which is explained by the size and the intrinsic disorder of the tubes [15]. To investigate the effect of treatment on random and dense vertical MWCNTs, contact angles are measured. Vertically aligned MWCNTs maintain a characteristic hydrophobicity (average contact angle: $107^{\circ}$ ), while randomly oriented tubes become hydrophilic after acid treatment (average contact angle: $24^{\circ}$ ). These results show that treated random nanotubes are the most suitable for biosensor applications. Values of the contact angles are reported in Table I.

TABLE I

CONTACT ANGLE MEASUREMENTS.

\begin{tabular}{cc}
\hline & Contact angle \\
\hline Random MWCNTs & \\
\hline Pristine & $114^{\circ} \pm 2^{\circ}$ \\
Acid treated & $24^{\circ} \pm 9^{\circ}$ \\
\hline Dense vertical MWCNTs \\
\hline Pristine & $109^{\circ} \pm 2^{\circ}$ \\
Acid treated & $107^{\circ} \pm 4^{\circ}$ \\
\hline
\end{tabular}

\section{Electrochemical characterization with ferricyanide}

The redox kinetics of potassium ferricyanide is studied by CV. Fig. 2 (a) displays voltammograms of $\left[\mathrm{Fe}(\mathrm{CN})_{6}\right]^{-3}$ / $\left[\mathrm{Fe}(\mathrm{CN})_{6}\right]^{-4}$ couple acquired at $100 \mathrm{mV} / \mathrm{s}$ in $5 \mathrm{mM}$ ferricyanide solution. In this conditions, the peak-to-peak separation $\left(\Delta E_{p}\right)$ is calculated in order to evaluate the reaction reversibility. For pristine random MWCNTs the separation between anodic and cathodic peaks is $259 \mathrm{mV}$. With the same orientation, but adding acid treatment, $\Delta E_{p}$ is $415 \mathrm{mV}$. The significant increase of $\Delta E_{p}$ proves that electron transfer to ferricyanide in solution is slower after treating randomly oriented MWCNTs. For dense vertical MWCNTs $\Delta E_{p}$ is 177 before and $254 \mathrm{mV}$ after treatment. The kinetics of reaction decreases also with dense vertical MWCNTs. According to the Laviron model [16], $k_{\text {et }}$ goes from $0.049 \pm 0.001$ to $0.010 \pm 0.001$ $\mathrm{s}^{-1}$ after acid activation. It is likely that the acid activation exposes more sidewalls especially for random MWNTs. As nanotube walls have similar electrochemical behavior to the graphite basal plane, a slow electron transfer rate appears
[10]. With randomly oriented MWCNTs the increase of $\Delta E_{p}$ is bigger $(159 \mathrm{mV})$ than for dense vertical nanotubes (77 $\mathrm{mV})$. Consequently, the the reaction reversibility decreases more for randomly oriented CNTs. From these results, we can assume that the treatment affects in particular nanotube sidewall structure.

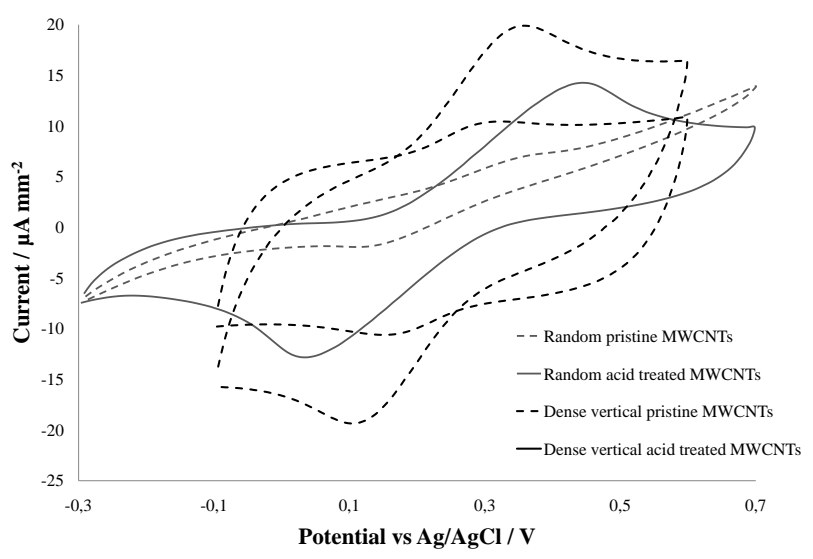

(a)

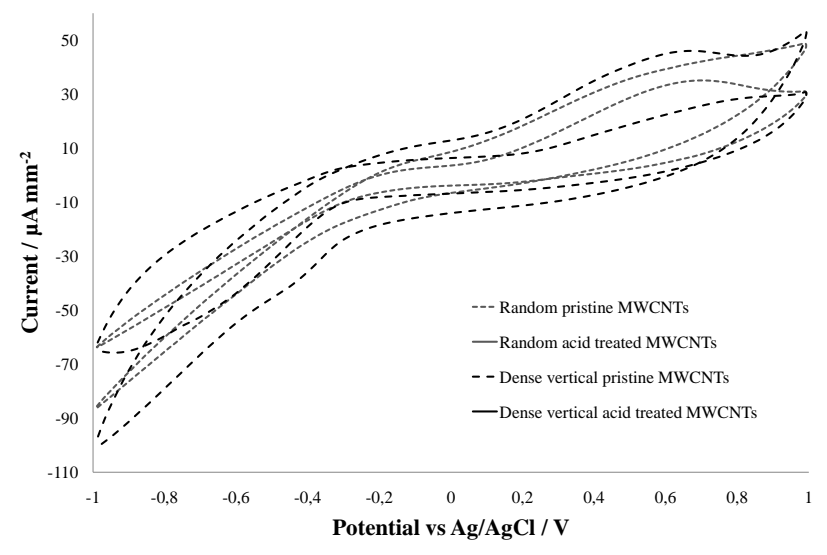

(b)

Fig. 2. Voltammograms obtained with pristine (dotted curve) and acid treated (solid curve) randomly oriented (gray curve) and vertically aligned (black curve) MWCNTs. Concentration: potassium ferricyanide $5 \mathrm{mM}(\mathrm{a})$ and hydrogen peroxide $30 \mathrm{mM}($ b). Scan rate: $100 \mathrm{mV} / \mathrm{s}$.

From Fig. 2 (a) it is possible to note that peaks are higher and well-shaped after acid treatment. This improvement is due to the increase of the electroactive area calculated by using equation 2. Pristine randomly oriented MWCNTs show a ratio between electroactive area and real area of 0.227 \pm 0.005 . Instead, the value related to the acid activated 
is one order of magnitude more $(1.16 \pm 0.02)$ due to the presence of active sites on CNT sidewall after the treatment. On the other hand, for dense vertical MWCNTs treatment causes a smaller increase of the electroactive area of about $34 \%$ (from $0.334 \pm 0.007$ to $0.506 \pm 0.010$ ). In vertically aligned CNTs the treatment does not act on CNT sidewalls because of their low accessibility. To confirm these results, sensibility and detection limit are calculated in reference to anodic peaks. For pristine random MWCNTs, sensitivity is one orders of magnitude smaller than for treated MWCNTs $\left(29.0 \pm 0.2\right.$ and $166.8 \pm 0.9 \mu \mathrm{A} /\left(\mathrm{mM} \mathrm{cm}^{2}\right)$ respectively). For untreated and treated vertical MWCNTs the sensitivity remain more or less constant $\left(133.0 \pm 0.7 \mu \mathrm{A} /(\mathrm{mM} \mathrm{cm})^{2}\right)$ and $126.5 \pm 0.7 \mu \mathrm{A} /\left(\mathrm{mM} \mathrm{cm}^{2}\right)$ respectively). Detection limit remains approximately unchanged by using vertical MWCNTs as electrode $\left(0.02 \mathrm{mM} \mathrm{cm}{ }^{2}\right.$ for pristine and $0.01 \mathrm{mM} \mathrm{cm}{ }^{2}$ for treated MWCNTs). On the contrary, for randomly oriented MWCNTs it goes from 0.16 to $0.02 \mathrm{mM} \mathrm{cm}$.

\section{Detection of hydrogen peroxide}

Hydrogen peroxide is also utilized as analyte, because it is a product of reactions catalyzed by many enzymes, like oxidases and peroxidases [9]. Also with hydrogen peroxide as target, the peaks are higher and well-shaped after acid treatment (see Fig. 2 (b)). For randomly oriented tubes sensitivity goes from $28.8 \pm 0.1 \mu \mathrm{A} /\left(\mathrm{mM} \mathrm{cm}^{2}\right)$ to $59.2 \pm 0.4 \mu \mathrm{A} /\left(\mathrm{mM} \mathrm{cm}^{2}\right)$ after the treatment. Detection limit does not show a significant change by using acid treatment passing from from 28.6 to 21.4 $\mu \mathrm{M} \mathrm{cm}{ }^{2}$. The different results obtained with the two probes are due to the specific electrochemical sensitivity of ferricyanide and hydrogen peroxide to various surface electrode states. Nonetheless, in both cases the acid activation causes a big increase of sensitivity using random MWCNTs.

EDX analysis is conducted to better understand the cause of the improved electrochemical results after the treatment for randomly oriented nanotubes. EDX reveals a chemical composition unchanged with the acid activation (data not shown). Therefore, treatment produces only structural defects and does not introduce any oxygen-contained functional groups. Consequently, defects are responsible of the improved sensitivity and detection limit at random treated MWCNTs [17].

\section{CONCLUSION}

The goal of the present paper is determining if the activation is more relevant on CNT walls or tips. For this aim, ferricyanide and hydrogen peroxide are used as target redox couples. The sensible change of wettability for randomly oriented nanotubes confirms the assumption that acid treatment causes a predominant change on CNT sidewalls. By using ferricyanide solutions, slowest kinetics characterizes acid-treated MWCNTs. The found behavior has some affinities with graphite basal plane [10]. Sensitivity, detection limit and electroactive area of treated random MWCNTs are in the same order of magnitude of those obtained with dense vertical MWCNTs. EDX analysis reveals the nature of the tube modification. As the atomic percentage of oxygen remains unchanged, we infer that acid treatment produces only edge-like-plane defects on CNT walls. Accordingly, structural defects are the cause of the big changes of sensitivity and detection limit values for random MWCNTs after the treatment [17].

In conclusion, acid treatment acts on the nanotube walls creating structural defects and improving the electrochemical properties. Using ferricyanide as target molecule, we have proved that randomly oriented MWCNTs after the treatment show values of sensitivity and detection limit of the same order of magnitude of the vertically aligned. Interestingly, electrochemical responses at the electrode surface depend on the type of target. The introduction of wall defects influences more nanotube-ferricyanide interaction rather than the interaction between MWCNTs and hydrogen peroxide. Considering the big increase of wettability on randomly oriented MWCNTs, we can argue that these systems are more suitable for biosensing applications compared to vertically packed.

\section{ACKNOWLEDGMENT}

The authors would like to thank Dr. M. Cantoni and Ms. F. Bobard for EDX analysis, S. Guastella to acquire FE-SEM images and $\mathrm{C}$. Boero for help in manuscript revision. The research has been supported by the i-IronIC project. The iIronIC project was financed with a grant from the Swiss NanoTera.ch initiative and evaluated by the Swiss National Science Foundation.

\section{REFERENCES}

[1] S. Carrara, A. Cavallini, A. Garg, and G. De Micheli. In IEEE/ICME International Conference on Complex Medical Engineering, 2009., 1-6, 2009.

[2] S. Carrara, V.V. Shumyantseva, A.I. Archakov, and B. Samorí. Biosensors and Bioelectronics, 24(1):148-150, 2008.

[3] S. Joseph, J.F. Rusling, Y.M. Lvov, T. Friedberg, and U. Fuhr. Biochemical pharmacology, 65(11):1817-1826, 2003.

[4] V. Erokhin G. De Micheli A. Cavallini, S. Carrara. In IEEE International Conference PRIME 2010, Berlin, 1-4, 18-21 July 2010.

[5] M. Musameh, N.S. Lawrence, and J. Wang. Electrochemistry Communications, 7(1):14-18, 2005.

[6] A.F. Holloway, G.G. Wildgoose, R.G. Compton, L. Shao, and M.L.H. Green. Journal of Solid State Electrochemistry, 12(10):1337-1348, 2008.

[7] K. Balasubramanian and M. Burghard. Analytical and Bioanalytical Chemistry, 385(3):452-468, 2006.

[8] K. Gong, S. Chakrabarti, and L. Dai. Angewandte Chemie International Edition, 47(29):5446-5450, 2008.

[9] G. Del Vecchio D. Albini L. Calz G. De Micheli C. Boero, S. Carrara. In IEEE/ICME International Conference on Complex Medical Engineering 2010, Gold Coast, Australia, 288-293, July 13-15, 2010.

[10] J. Li, A. Cassell, L. Delzeit, J. Han, and M. Meyyappan. J. Phys. Chem. B, 106(36):9299-9305, 2002.

[11] M. Kumar and Y. Ando. Diamond and Related Materials, 12(1011):1845-1850, 2003.

[12] M. Kumar and Y. Ando. In Journal of Physics: Conference Series, volume 61, page 643. IOP Publishing, 2007.

[13] S. Porro, S. Musso, M. Giorcelli, A. Chiodoni, and A. Tagliaferro. Physica E: Low-dimensional Systems and Nanostructures, 37(1-2):1620, 2007.

[14] Y.S. Chen, J.H. Huang, and C.C. Chuang. Carbon, 47(13):3106-3112, 2009.

[15] M. Giorcelli. Ph.D. Thesis, 2008.

[16] E. Laviron. Journal of Electroanalytical Chemistry, 101(1):19-28, 1979.

[17] C. Hu and S. Hu. Langmuir, 24(16):8890-8897, 2008. 